\section{Commentary: When a low profile means a high impact}

\author{
David Blitzer, MD, ${ }^{\mathrm{a}}$ Alvise Guariento, $\mathrm{MD},{ }^{\mathrm{b}}$ and \\ Vladimiro Vida, MD $^{\mathrm{b}}$
}

Szeto and colleagues ${ }^{1}$ report 1-year results for the RelayPro (Terumo Aortic), a new low-profile iteration of the thoracic endograft for application in descending thoracic aortic aneurysms and in penetrating atherosclerotic ulcers. After application in 110 patients with diverse pathologies in centers in the United States and Japan, the authors report a $6.4 \%$ rate of major adverse events that included postprocedural stroke, procedural blood loss $>1000 \mathrm{~mL}$, paralysis events, and renal failure. Overall clinical results were compared favorably with studies from prior iterations of the Relay endograft system. ${ }^{2}$

Perhaps more importantly, the authors report a $100 \%$ technical success rate with no incidence of loss of stentgraft patency, rupture, fracture, or migration. Although the Japanese centers utilized only surgical cutdown for deployment, the US centers were able to perform percutaneous deployment in $73.5 \%$ of cases. These technical components are particularly important in this study because the major advantage afforded by the RelayPro system is the lower profile delivery mechanism that is meant to facilitate percutaneous deployment of the graft and also to enable the application of this technology in ileofemoral arteries that are smaller or more diseased.

Progression to a lower profile sheath over time, as Szeto and colleagues ${ }^{1}$ point out, has not been without complications or hurdles because manufacturers have sought to reduce the profile of the endograft systems while

\footnotetext{
From the a Department of Surgery, Columbia University Medical Center, New York, NY; and ${ }^{b}$ Pediatric Cardiac Surgery, Department of Cardiac, Thoracic, Vascular Sciences, and Public Health, University of Padua, Padua, Italy.

Disclosures: The authors reported no conflicts of interest.

The Journal policy requires editors and reviewers to disclose conflicts of interest and to decline handling or reviewing manuscripts for which they may have a conflict of interest. The editors and reviewers of this article have no conflicts of interest.

Received for publication Nov 9, 2021; revisions received Nov 9, 2021; accepted for publication Nov 9, 2021; available ahead of print Nov 12, 2021.

Address for reprints: Alvise Guariento, MD, Pediatric Cardiac Surgery, Department of Cardiac, Thoracic, Vascular Sciences, and Public Health, University of Padua, Via Giustiniani 2, 35100 Padua, Italy (E-mail: alvise.guariento@unipd.it).

J Thorac Cardiovasc Surg 2022;163:1751-2

$0022-5223 / \$ 36.00$

Copyright (c) 2021 by The American Association for Thoracic Surgery

https://doi.org/10.1016/j.jtcvs.2021.11.016
}

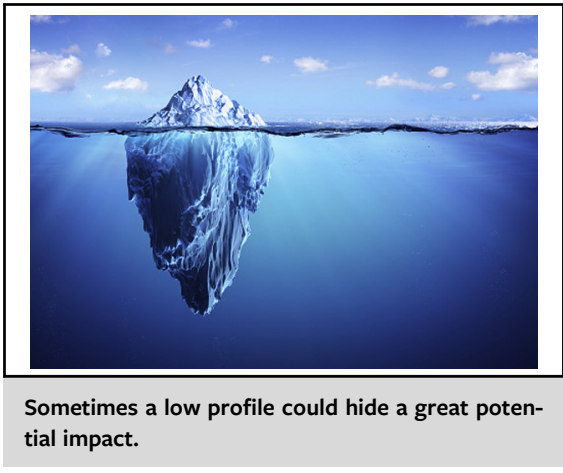

CENTRAL MESSAGE

A new, low-profile thoracic endograft system has been successfully applied to a wide array of patients, expanding the pool of those who can benefit from this treatment modality.

maintaining good clinical outcomes, which is of primary importance. They point out the success of the RelayPro system to reduce the profile of the graft material, the radiopaque marker, and the outer and inner sheaths. The first iteration of the Relay endograft was approved by the Food and Drug Administration in 2012 and it took the following 9 years to reach this point in its technical advancement. The evolution translates to better procedural characteristics for patients with smaller ileofemoral vessels,

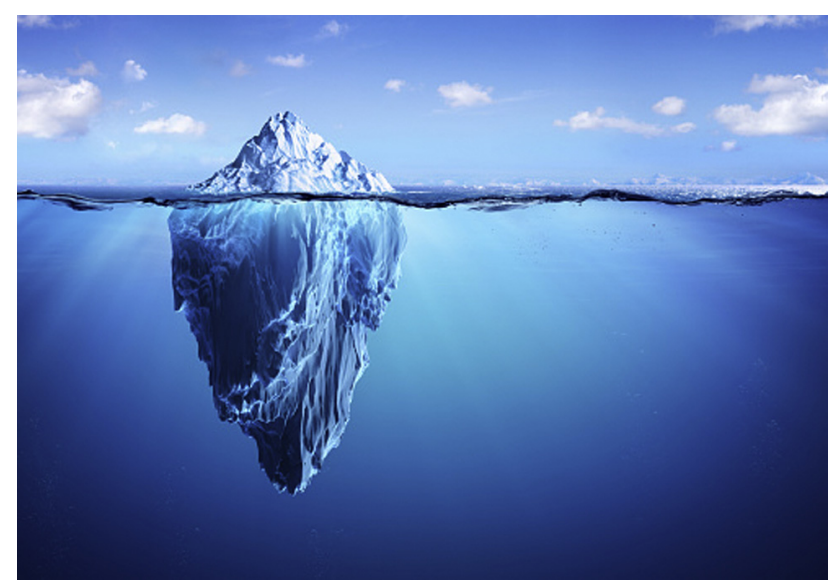

FIGURE 1. A low-profile system could be highly influential for a wide variety of patients. 
particularly women. Other studies, particularly with transcatheter aortic valve replacement, have demonstrated why the investigation of sex-based differences in procedural outcomes is critical. ${ }^{3,4}$ Although the first publication of results with the Relay device featured gender parity with $52.6 \%$ being men, of the 26 patients who required iliac conduits in that study, $21(80.8 \%)$ were women, with 1 woman ultimately being deemed anatomically unsuitable. ${ }^{2}$ Conduits were only required in 2 patients $(2.9 \%)$ with the RelayPro and technical success was achieved in $100 \%$ of patients.

This all goes to show that the constant evolution of medical technologies is not only important for the goal of continuous improvement in clinical outcomes in general, but also for the principle of providing high-quality care to the wide variety of patients that we treat, regardless of sex, age, or race (see Figure 1).

\section{References}

1. Szeto WY, Vallabhajosyula P, Matsuda H, Moainie SL, Sharafuddin MJ, Corvera J, et al. One-year results with a low-profile endograft in subjects with thoracic aortic aneurysm and ulcer pathologies. J Thorac Cardiovasc Surg. 2022;163:1739-50.e4.

2. Farber MA, Lee WA, Szeto WY, Panneton JM, Kwolek CJ. Initial and midterm results of the Bolton Relay Thoracic Aortic Endovascular Pivotal Trial. J Vasc Surg. 2017;65:1556-66.e1. https://doi.org/10.1016/j.jvs.2016.11.061

3. Chandrasekhar J, Dangas G, Yu J, Vemulapalli S, Suchindran S, Bora AN, et al. Sex-based differences in outcomes with transcatheter aortic valve therapy: TVT Registry from 2011 to 2014. J Am Coll Cardiol. 2016;68:2733-44. https: //doi.org/10.1016/j.jacc.2016.10.041

4. Williams M, Kodali SK, Hahn RT, Humphries KH, Nkomo VT, Cohen DJ, et al. Sex-related differences in outcomes after transcatheter or surgical aortic valve replacement in patients with severe aortic stenosis: insights from the partner trial (placement of aortic transcatheter valve). J Am Coll Cardiol. 2014;63:1522-8. https://doi.org/10.1016/j.jacc.2014.01.036

\section{Commentary: Profile matters: 1-year results of low profile endograft with thoracic aortic aneurysm and ulcer pathologies}

\author{
Sashi K. Inkollu, MD, ${ }^{\mathrm{a}}$ and \\ Carlos F. Bechara, MD, DFSVS ${ }^{b}$
}

Some of the most common complications encountered in treating thoracic aortic pathologies are related to vascular access such as bleeding from failure of percutaneous access closure devices and iliac rupture as

From the ${ }^{a}$ Department of Vascular Surgery, Marshfield Clinic Health Systems, Marshfield, Wis; and ${ }^{\mathrm{b}}$ Department of Vascular Surgery, Loyola University Medical Center, Maywood, Ill.

Disclosures: Dr Bechara is a consultant and speaker for Gore Medical, Cook Medical, Terarecon, and Penumbra, owns stock in Mokita Medical, and is a consultant (CEC) for Endospan. Dr Inkollu reported no conflicts of interest.

The Journal policy requires editors and reviewers to disclose conflicts of interest and to decline handling or reviewing manuscripts for which they may have a conflict of interest. The editors and reviewers of this article have no conflicts of interest.

Received for publication Nov 16, 2021; revisions received Nov 16, 2021; accepted for publication Nov 17, 2021; available ahead of print Nov 20, 2021.

Address for reprints: Sashi K. Inkollu, MD, Department of Vascular Surgery, Marshfield Clinic Health Systems, 1000 N Oak Ave, Suite 3C, Marshfield, WI 54449 (E-mail: inkollu.sashi@marshfieldclinic.org).

J Thorac Cardiovasc Surg 2022;163:1752-3

$0022-5223 / \$ 36.00$

Copyright (c) 2021 by The American Association for Thoracic Surgery

https://doi.org/10.1016/j.jtcvs.2021.11.030

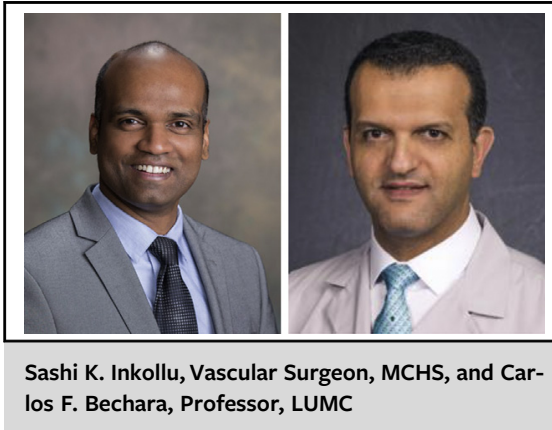

CENTRAL MESSAGE

The newer-generation RelayPro thoracic device (Terumo Corp) offers a lower profile and better conformability but will its longterm results hold good for device integrity?

well as access vessel occlusion from dissection and thrombosis. These complications are more common in the presence of smaller-access vessels relative to the device profile such as in women or in the presence of occlusive disease. Szeto and colleagues ${ }^{1}$ have presented the 1-year results of the new-generation low profile RelayPro thoracic aortic stent (Terumo Corp) graft in the treatment of aneurysm and ulcer pathologies. This was a prospective, multicenter and international study of 